SV

\title{
AIR LOADS ON A RIGID PLATE OSCILLATING NORMAL TO A FIXED SURFACE
}

\author{
W. M. Beltman, P. J. M. van der Hoogt, R. M. E. J. Spiering and \\ H. TIJDEMAN \\ University of Twente, Department of Mechanical Engineering, P.O. Box 217, \\ 7500 AE Enschede, The Netherlands
}

(Received 20 February 1995, and in final form 18 April 1997)

\begin{abstract}
This paper deals with a theoretical and experimental investigation on a rigid, rectangular plate oscillating in the proximity of a fixed surface. The plate is suspended by springs. The airloads generated by the oscillating motion of the plate are determined. Due to the fact that the plate is rigid, the system is modelled as a 1-DOF system. The influence of the surrounding air is detected by changes in the plate's natural frequency and damping. For the behaviour of the air in the gap between the plate and the fixed surface an analytical solution is presented. This solution includes the effects of inertia, viscosity, compressibility and thermal conductivity. It is shown that the main parameters governing the motion of the air in the gap are the shear wave number, the reduced frequency, the narrowness of the gap and the aspect ratio of the plate. With these parameters the validity of several simplifications can easily be demonstrated and solutions, given in the literature, can be put in perspective. Special experiments were carried out with an oscillating solar panel in order to verify the analytical model. The analytical results and the experimental results show fair agreement. The solutions shows that for low shear wave numbers the effects of viscosity cannot be discarded.

(C) 1997 Academic Press Limited
\end{abstract}

\section{INTRODUCTION}

This paper deals with the air loads on a rigid rectangular plate oscillating normal to a fixed surface. These forces can be interpreted as an added mass, added stiffness and added damping for the oscillating plate. For the behaviour of the air in the gap between the plate and the fixed surface an analytical solution is presented. The solution includes the effects of inertia, viscosity, compressibility and thermal conductivity. The solution is written in terms of dimensionless parameters that govern the behaviour of the air. The advantage of this approach is that, in contrast with previous publications in this area, the range of validity can be determined relatively easy and the validity of several simplifications can be justified. After an overview of the literature on the subject [1-29], the analytical solution is discussed. Special attention is paid to the effects of viscosity and thermal conductivity. In order to compare the results from the analytical solution with the results from a standard technique, based on the wave equation, finite element calculations were carried out. In these calculations the air is represented as a compressible, inviscid medium. The finite element results can directly be compared with the analytical results when the effects of viscosity and thermal conductivity are ruled out in the analytical model. Finally, an experimental set-up is discussed. This set-up is used to measure the eigenfrequency and the damping of the system. The experiments, described in the literature, all deal with very small gap widths, e.g., $0 \cdot 1 \mathrm{~mm}$. For these gaps widths low but significant damping levels in the frequency range of interest were found. However, such a small gap width is difficult 
to control and consequently the accuracy of the results is often not very clear. A further drawback is the fact that the amplitude of oscillation has to be kept very small in order to avoid non-linearities.

The experimental set-up presented in this paper was specially designed to avoid many of these problems. With the help of the aforementioned dimensionless parameters a large scale set-up was constructed. Typical properties of the set-up are as follows: plate dimensions $0.98 \times 0.98 \mathrm{~m}$, gap widths $3-650 \mathrm{~mm}$ and a frequency range from $1-10 \mathrm{~Hz}$. With this set-up damping values up to almost critical damping were measured with satisfying accuracy. Calculations and experiments show good agreement.

\section{FORMULATION OF THE PROBLEM}

A rigid, rectangular plate, is considered suspended on springs attached to the corners of the plate (see Figure 1). The plate is located parallel to a fixed surface and performs small harmonic oscillations normal to the surface. The distance between the plate and the fixed surface, $\bar{h}(t)$ is given by

$$
\bar{h}(t)=h_{0}\left[1+h \mathrm{e}^{\mathrm{i} \omega t}\right],
$$

where $h_{0}$ is the mean distance between the plate and the fixed surface, $h$ is the dimensionless amplitude of the oscillation, $\omega$ is the angular frequency and $t$ refers to time. (A list of symbols is given in Appendix E.)

The $x$ and the $y$ co-ordinates are the in-plane co-ordinates; the $z$ direction is the direction normal to the fixed surface. The origin is located on the fixed surface at the centre of the plate. The plate has dimensions of $2 l_{x}$ in the $x$ direction and $2 l_{y}$ in the $y$ direction. The co-ordinates are made dimensionless according to:

$$
x=\bar{x} / l_{x}, \quad y=\bar{y} / l_{y}, \quad z=\bar{z} / h_{0} .
$$

It is also possible to introduce dimensionless co-ordinates with respect to the acoustic wave length, i.e., $x=\omega \bar{x} / c_{0}, y=\omega \bar{y} / c_{0}$. The appropriate form of the "narrow gap" equation (see section 4$)$ is then valid for $w / u \ll 1, w / v \ll 1, k \ll 1$ and $k / s \ll 1$. Evidently, both methods finally result in the same expression for the pressure distribution in the gap. In this investigation the approach with non-dimensional co-ordinates based on the plate dimensions is preferred, since it enables a direct connection to be made with the dimensionless form of the differential equation which governs the behaviour of a flexible plate.

The forces acting on the plate due to the pressure distribution in the gap are calculated. The plate is assumed to be rigid. Therefore any uncertainty with respect to the dynamical behaviour of the plate is excluded. The boundary condition $p=0$ is applied (see Figure 2). This is a simplification, since the pressure distribution outside the gap will be affected by the pressure distribution in the gap and vice versa. For narrow gaps, however, the magnitude of the pressure perturbation in the gap is very large compared to the magnitude

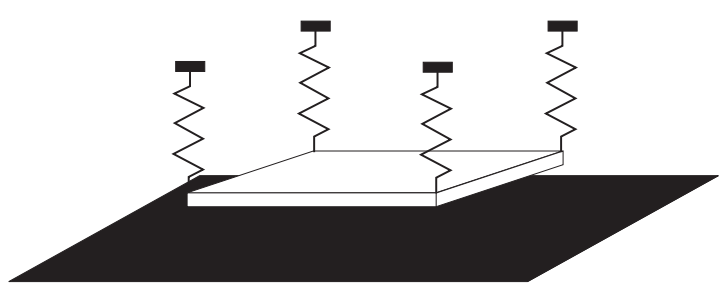

Figure 1. Oscillating plate. 


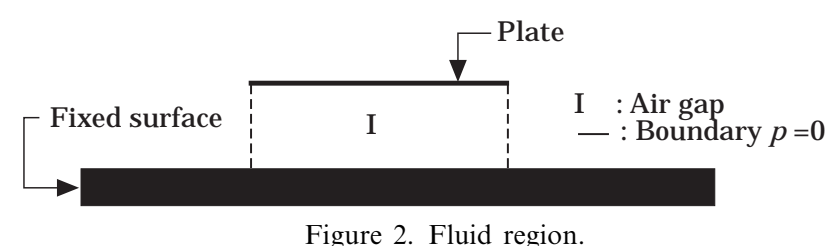

Figure 2. Fluid region.

of the pressure perturbation outside the gap. Therefore the boundary condition $p=0$ is a realistic assumption, as is confirmed by straightforward potential flow calculations and a comparison between numerical and experimental results.

\section{OVERVIEW}

The basic equations for the behaviour of the air in the gap are derived in section 3.1. The equations are written in a dimensionless form. The resulting dimensionless parameters characterize the behaviour of the air. The advantage of this approach is that, in contrast with previous publications in this area, the validity of solutions can be determined relatively easy. The parameters are used in section 3.2 to compare the various solutions given in the literature.

\subsection{BASIC EQUATIONS}

The motion of the air in the gap is governed by the following equations: the Navier Stokes equations, the equation of continuity, the equation of state for an ideal gas and the energy equation. An approach similar to that of Tijdeman [2] is followed, resulting in the introduction of a number of assumptions: no internal heat generation; homogeneous medium, which implies that the gap width and the wave length must be large in comparison with the mean free path-for air at normal atmospheric conditions this assumption breaks down for $h_{0}<10^{-7} \mathrm{~m}$ and $f>10^{8} \mathrm{~Hz}$; no mean flow; small, sinusoidal perturbations; no circulation and no turbulence.

The steady state condition is characterized by a zero mean velocity, a mean pressure $p_{0}$, a mean density $\rho_{0}$ and a mean temperature $T_{0}$. The undisturbed speed of sound $c_{0}$ is used to non-dimensionalize the velocities. Small dimensionless perturbations $(u, v, w, p, \rho$ and $T$ ) upon the steady state condition are introduced:

$$
\begin{gathered}
\bar{u}=c_{0} u(\bar{x}, \bar{y}, \bar{z}) \mathrm{e}^{\mathrm{i} \omega t}, \quad \bar{p}=p_{0}\left[1+p(\bar{x}, \bar{y}, \bar{z}) \mathrm{e}^{\mathrm{i} \omega t}\right], \quad \bar{v}=c_{0} v(\bar{x}, \bar{y}, \bar{z}) \mathrm{e}^{\mathrm{i} \omega t}, \\
\bar{\rho}=\rho_{0}\left[1+\rho(\bar{x}, \bar{y}, \bar{z}) \mathrm{e}^{\mathrm{i} \omega t}\right], \quad \bar{w}=c_{0} w(\bar{x}, \bar{y}, \bar{z}) \mathrm{e}^{\mathrm{i} \omega t}, \quad \bar{T}=T_{0}\left[1+T(\bar{x}, \bar{y}, \bar{z}) \mathrm{e}^{\mathrm{i} \omega t}\right] .
\end{gathered}
$$

The basic equations are linearized and written in a dimensionless form. A set of six linear differential equations is obtained. Together with the appropriate boundary conditions these equations suffice to determine all unknown quantities. Details of the derivation of these equations are given in Appendix A. The resulting linearized equations are written as

$$
\begin{gathered}
\mathrm{i} u=-\frac{g}{k} \frac{1}{\gamma} \frac{\partial p}{\partial x}+\frac{1}{s^{2}}\left[g^{2} \frac{\partial^{2} u}{\partial x^{2}}+\left(\frac{g}{a}\right)^{2} \frac{\partial^{2} u}{\partial y^{2}}+\frac{\partial^{2} u}{\partial z^{2}}\right]+\frac{1}{3} \frac{g}{s^{2}} \frac{\partial}{\partial x}\left[g \frac{\partial u}{\partial x}+\left(\frac{g}{a}\right) \frac{\partial v}{\partial y}+\frac{\partial w}{\partial z}\right], \\
\mathrm{i} v=-\frac{g}{a k} \frac{1}{\gamma} \frac{\partial p}{\partial y}+\frac{1}{s^{2}}\left[g^{2} \frac{\partial^{2} v}{\partial x^{2}}+\left(\frac{g}{a}\right)^{2} \frac{\partial^{2} v}{\partial y^{2}}+\frac{\partial^{2} v}{\partial z^{2}}\right]+\frac{1}{3} \frac{g}{a s^{2}} \frac{\partial}{\partial y}\left[g \frac{\partial u}{\partial x}+\left(\frac{g}{a}\right) \frac{\partial v}{\partial y}+\frac{\partial w}{\partial z}\right],
\end{gathered}
$$




$$
\begin{gathered}
\mathrm{i} w=-\frac{1}{k} \frac{1}{\gamma} \frac{\partial p}{\partial z}+\frac{1}{s^{2}}\left[g^{2} \frac{\partial^{2} w}{\partial x^{2}}+\left(\frac{g}{a}\right)^{2} \frac{\partial^{2} w}{\partial y^{2}}+\frac{\partial^{2} w}{\partial z^{2}}\right]+\frac{1}{3} \frac{1}{s^{2}} \frac{\partial}{\partial z}\left[g \frac{\partial u}{\partial x}+\left(\frac{g}{a}\right) \frac{\partial v}{\partial y}+\frac{\partial w}{\partial z}\right], \\
g \frac{\partial u}{\partial x}+\left(\frac{g}{a}\right) \frac{\partial v}{\partial y}+\frac{\partial w}{\partial z}=-\mathrm{i} k \rho, \quad p=\rho+T \\
\mathrm{i} T=\frac{1}{s^{2} \sigma^{2}}\left[g^{2} \frac{\partial^{2} T}{\partial x^{2}}+\left(\frac{g}{a}\right)^{2} \frac{\partial^{2} T}{\partial y^{2}}+\frac{\partial^{2} T}{\partial z^{2}}\right]+\mathrm{i}\left[\frac{\gamma-1}{\gamma}\right] p
\end{gathered}
$$

where

$s=h_{0} \sqrt{\rho_{0} \omega / \mu}$ shear wave number, $\quad \sigma=\sqrt{\mu C_{p} / \lambda}$ square root of the Prandtl number,

$$
k=\omega h_{0} / c_{0} \text { reduced frequency, }
$$

$\gamma=C_{p} / C_{v}$ ratio of specific heats, $g=h_{0} / l_{x}$ narrowness of the gap,

$$
a=l_{y} / l_{x} \text { aspect ratio of the plate. }
$$

The dimensionless parameters, specified in equations (10), characterize the behaviour of the air in the gap. The values for $\sigma$ and $\gamma$ can be regarded as constants for a specific gas, e.g., air. The parameters $g$ and $a$ are geometry dependent. The aspect ratio of the plate is assumed to be equal to or larger than one: $a \geqslant 1$, according to a proper choice of the $x$ and $y$ directions. The two main parameters are the shear wave number, $s$, (sometimes also referred to as the Stokes number) and the reduced frequency, $k$. The shear wave number is a measure of the ratio between inertial and viscous forces. Hence, the shear wave number is an important quantity that governs the validity of several simplifications. The reduced frequency is a measure of the ratio between the gap width and the acoustic wave length.

\subsection{REVIEW OF SOLUTIONS GIVEN IN THE LITERATURE}

The problem of a plate vibrating in the direction normal to a fixed surface has been the subject of investigation in several scientific disciplines. In fluid mechanics the research was mainly aimed at the calculation of the steady streaming generated by the vibrating plate. This subject is strongly related to the well-known squeeze film damping in tribology. Tribologists have investigated squeeze film damping for more than a century. More recent research on this subject was initiated in the field of acoustics. A short overview of the research done in the mentioned disciplines will be given. The overview is summarized in Table 1. As far as possible, for the experiments the test range in terms of the shear wave number is indicated.

\subsubsection{Fluid mechanics}

When the plate performs lateral oscillations in its own plane the behaviour is governed by the linear equations of parallel flow. The solution for this problem was given by Lamb in 1932 [4]. When the plate oscillates normal to its own plane no exact solutions of the full unsteady Navier-Stokes equations are known to the present authors. Several publications deal with simplified situations. Kuhn and Yates [5] were the first to include inertial terms but they still ignored the second order in-plane velocity derivatives for the viscous part in the Navier-Stokes equations. Terrill [6] considered the full Navier-Stokes problem. He used a perturbation method in order to obtain a solution and expanded the solution in terms of the small amplitude of oscillation. 


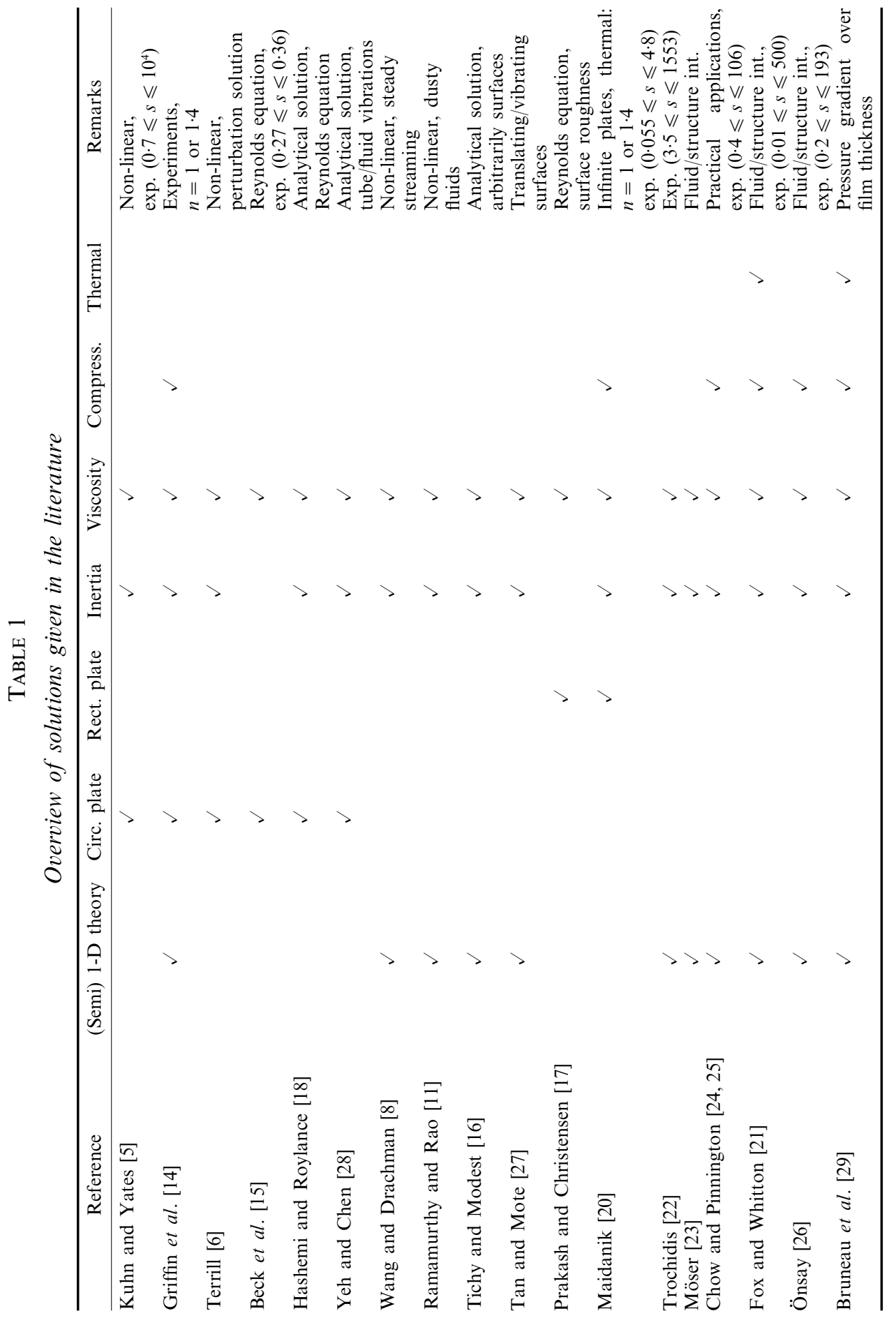


Ishizawa [7] used the same basic equations but he expanded the solution in terms of a modified Reynolds number. This modified Reynolds number is related to the shear wave number $s$ according to:

“Modified Reynolds number" $\equiv h_{0}^{2} \rho_{0} \omega / \mu=s^{2}$

Ishizawa pursued a perturbation to second order and obtained the steady streaming. The first order terms are purely oscillatory and have a zero mean value. The second order terms, however, are composed of an oscillatory flow of twice the basic frequency and a steady part. The steady part induces a mean lift acting on the plate. The paper by Wang and Drachman [8] describes a method to calculate the motion of the fluid when the plate performs both normal and lateral oscillations. Their investigations showed that the steady lift depends on the normal oscillation only. Ramamurthy and Rao [9] extended the work of Wang and Drachman to fluids with dust particles.

\subsubsection{Tribology}

The behaviour of a squeezed film between two surfaces was first studied by Stefan in 1874 [10]. He considered the case of two rigid circular plates performing out of phase normal oscillations. In 1886 Reynolds [11] extended this work to arbitrarily shaped surfaces. Reynolds' lubrication theory has been used in a large number of problems. In his theory the inertial terms are neglected: due to the high rate of shear the inertial forces are assumed to be negligible compared to the viscous forces. In the last decades, however, increasing machine speeds and low viscosity lubricants have necessitated the inclusion of inertial effects. The developments of gas bearings for instance demanded a profound knowledge of the inertial effects.

Kahlert [12] and Brand [13] were the first who recognized that the importance of inertia depends upon the "modified Reynolds number", as specified in the previous section on fluid mechanics. They demonstrated that inertial terms become significant when the Reynolds number is of order unity or higher. Since then, a lot of research has been done on the influence of inertia. Besides the modified Reynolds number two other dimensionless parameters appear in the tribology research: the "Sommerfeld squeeze number" and the "squeeze velocity number". Both parameters can be expressed in terms of the parameters specified in section 3.1:

$$
\begin{aligned}
& \text { “Sommerfeld squeeze number" } \equiv \mu \omega / p_{0}=\gamma k^{2} / s^{2} \\
& \text { "Squeeze velocity number" } \equiv \rho_{0} \omega^{2} h_{0}^{2} / p_{0}=\gamma k^{2} .
\end{aligned}
$$

There is a strong connection between tribology and fluid mechanics. Therefore it is hard to judge whether some research should be addressed as tribology or fluid mechanics research.

\subsubsection{Acoustics}

An important aspect in the field of acoustics concerns the calculation of fluid-structure interaction. In such calculations the influence of the surrounding fluid, in most cases air, on the vibrating structure is taken into account. Usually the fluid is modelled as compressible, inviscid medium with no mean flow. Under these conditions the behaviour of the fluid can be represented with the wave equation. When thin fluid layers however are trapped between surfaces of vibrating structures, the viscosity can no longer be neglected.

The damping of gas films was experimentally investigated by Ungar and Carbonell [19]. An explanation for the phenomena observed in their experiments was given in the theory 
of Maidanik [20]. He found that the damping was caused by the pumping of the gas. $\mathrm{He}$ applied a statistical approach of the structural wave field for high frequencies.

The more recent research in this field can be divided in three groups. The first group is concerned with the calculation of the dynamical behaviour of long, thin plates backed by a thin fluid layer. In 1980 Fox and Whitton [21] developed a method to calculate the damping of structural vibrations by thin gas films for the lower frequency modes. Their fluid model included the effects of inertia, viscosity, compressibility and thermal conductivity. Recently, Önsay [26] used this model to investigate the effect of the fluid layer thickness on the vibrational behaviour of a plate-fluid system. Both Fox and Whitton and Önsay performed a number of experiments in order to validate their calculations.

The second group is concerned with the dynamical behaviour of infinite plates, separated by a thin fluid layer. Trochidis [22] presented a report concerning the damping of flexible plates. In his calculations the plates were assumed to be of infinite length. Furthermore, it was assumed that the deformation of the plates can be regarded as one-dimensional: the displacements vary in one direction only. The importance of viscous effects was illustrated with a number of experiments. Möser [23] used a similar model, but he also included the effects of compressibility in his model with infinite plates. The practical application of squeeze film damping with air was investigated by Chow and Pinnington [24]. They developed an impedance method in order to calculate the loss factor of two infinite plates, separated by a thin gas layer. In 1988 they extended this theory to calculate the loss factor of a plate-liquid system [25]. For the high frequency range a statistical energy approach (SEA) was applied. The methods were used to develop configurations with high loss factors. Calculations and experiments showed that a significant amount of damping could be achieved with the squeeze film damping mechanism.

The third group is concerned with the interaction between a vibrating membrane and a thin layer of fluid. A recent investigation in this area was presented by Bruneau, Bruneau and Hamery [29]. In this investigation a model is presented which describes the behaviour of thin fluid films trapped between a vibrating membrane and a backing wall. As a starting point linearized equations are used, including the effects of thermal conductivity and viscosity. The model allows for a pressure gradient across the film thickness. The solution however is not written in a non-dimensional form. Furthermore it is difficult to extend their solution technique, used to describe the interaction with a vibrating membrane, to the interaction with vibrating plates.

\section{THE "NARROW GAP" SOLUTION}

\section{1. "NARROW GAP” SOLUTION}

When the gap width is small in comparison with the dimensions of the plate, and the velocity $w$ is negligible with respect to the in-plane velocities $u$ and $v$ (i.e., $g \ll 1, g / s \ll 1$, $w / v \ll 1$ and $w / u \ll 1$ ), the basic equations (4-9) of section 3.1 can be simplified to:

$$
\begin{array}{r}
\mathrm{i} u=-\frac{g}{k} \frac{1}{\gamma} \frac{\partial p}{\partial x}+\frac{1}{s^{2}} \frac{\partial^{2} u}{\partial z^{2}}, \quad \mathrm{i} v=-\frac{g}{a k} \frac{1}{\gamma} \frac{\partial p}{\partial y}+\frac{1}{s^{2}} \frac{\partial^{2} v}{\partial z^{2}}, \quad 0=-\frac{1}{k} \frac{1}{\gamma} \frac{\partial p}{\partial z}, \\
g \frac{\partial u}{\partial x}+\left(\frac{g}{a}\right) \frac{\partial v}{\partial y}+\frac{\partial w}{\partial z}=-\mathrm{i} k \rho, \quad p=\rho+T, \quad \mathrm{i} T=\frac{1}{s^{2} \sigma^{2}} \frac{\partial^{2} T}{\partial z^{2}}+\mathrm{i}\left[\frac{\gamma-1}{\gamma}\right] p .
\end{array}
$$

This set of equations can be rewritten as the equations derived by Fox and Whitton [21], which were not presented in a dimensionless form. In their analysis however they did 
conclude that the importance of inertia and viscosity depends on a dimenionless parameter $(\theta)$.

This quantity is in fact directly related to the shear wave number according to

$$
\theta \equiv h_{0} \sqrt{\rho_{0} \omega / 2 \mu}=\frac{1}{2} \sqrt{2} s .
$$

Equation (15) shows that the pressure perturbation $p$ is constant across the gap width for narrow gaps. The set of equations can be solved analytically. A detailed derivation of the solution can be found in Appendix B. The solution for the dimensionless pressure perturbation $p$ is written as

$$
p=\frac{4}{\pi} \frac{1}{g^{2}} \frac{k^{2} \gamma h}{B(s)} \sum_{q=1,3,5 . .}^{\infty} \frac{1}{q} \frac{(-1)^{(q-1) / 2}}{D^{2}}\left[1-\frac{\cosh (D x)}{\cosh (D)}\right] \cos \left(\frac{q \pi}{2} y\right),
$$

where

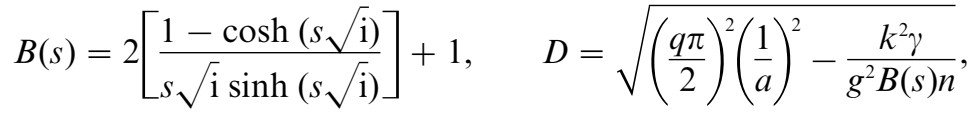

$$
\begin{aligned}
& n=\left[1-\left[\frac{\gamma-1}{\gamma}\right] B(s \sigma)\right]^{-1} .
\end{aligned}
$$

The same result is obtained if, instead of the energy equation and the equation of state, the following polytropic relation is used:

$$
\bar{p} / \bar{\rho}^{n}=\text { constant } .
$$

The polytropic constant, $n$, relates pressure and density. Evidently, the value of the parameter $n$, as given in equation (23), now accounts for the thermal effects. A detailed discussion on this constant is given in the next section.

\subsection{MAIN RESULTS OF THE "NARROW GAP" SOLUTION}

The "narrow gap" solution is based on the assumptions specified in section 3.3. In Appendix $\mathrm{C}$ it is shown that for large shear wave numbers the "narrow gap" solution reduces to a modified form of the wave equation: viscous forces can be neglected. For low shear wave numbers a linearized version of the Reynolds equation is obtained (see Appendix D): inertial forces can be neglected. The main characteristics of the narrow gap solution are summarized in Figure 3.

\subsubsection{In-plane velocity profile}

The transition from viscous dominated flow to inertial dominated flow can be illustrated with the in-plane velocity profile. The shape of this profile depends on the value of the shear wave number. In Figure 4 the magnitude of the velocity in the $x$ direction across the gap is given for different shear wave numbers. For high values a plane velocity profile is obtained. For very low shear wave numbers viscous forces dominate and the velocity profile reduces to the parabolic Poiseuille profile.

\subsubsection{Thermal effects}

The equation of state and the energy equation can be combined in the form of the polytropic relation (strictly speaking only in a layer-integrated sense, see Appendix B). The influence of the thermal conductivity is found in expression (23) for the polytropic constant 


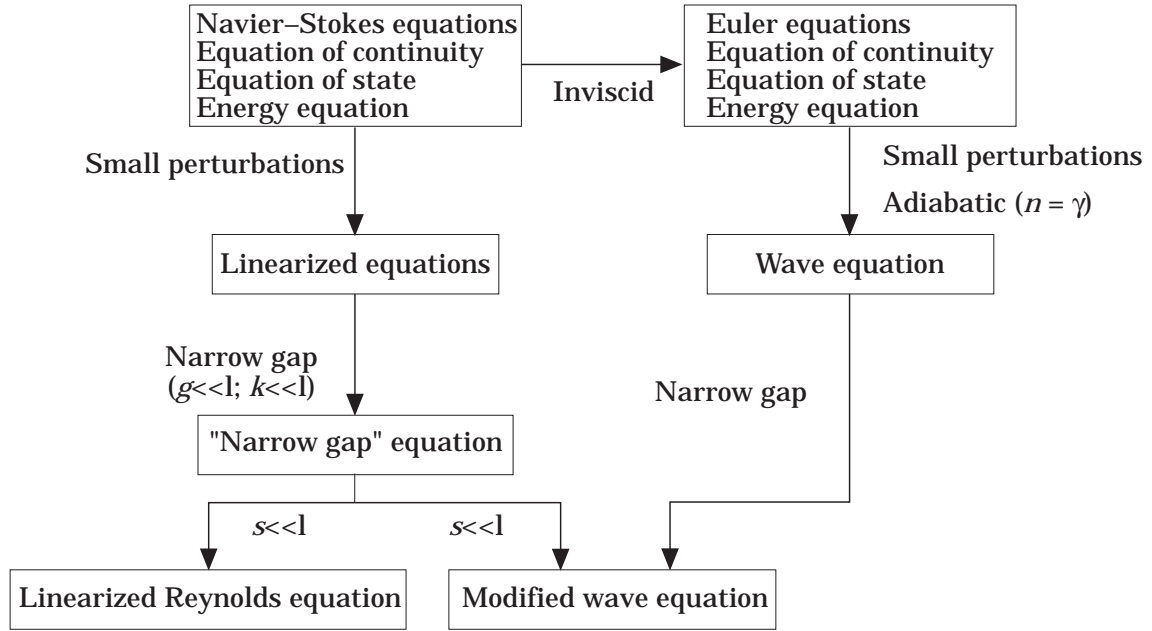

Figure 3. Main characteristics of the "narrow gap" solution.

$n$. This constant is a function of the product of the shear wave number and the square root of the Prandtl number. The value of the constant $n$ is influenced only by the thermal conductivity of the air, because the product $s \sigma$ does not contain the viscosity $\mu$. As can be seen from the definition of the function $B(s)$, the polytropic constant generally will be a complex quantity. This implies that there is a phase shift between the pressure perturbation $p$ and the density perturbation $\rho$. The value of $n$ as a function of the product $s \sigma$ is depicted in Figures 5 and 6. It can be seen that for low values of $s \sigma$ the polytropic constant reduces to 1 (Reynolds equation), which implies that the process occurs isothermally. For high values of $s \sigma$ the process however occurs isentropically (adiabatically) and $n$ takes the value of $\gamma$ (wave equation).

\section{DYNAMICAL BEHAVIOUR}

\subsection{MOBILITY FUNCTION}

The plate is assumed to be rigid, to move only in the $z$ direction and to remain parallel to the fixed surface. The system can therefore be regarded as a single-degree-of-freedom-

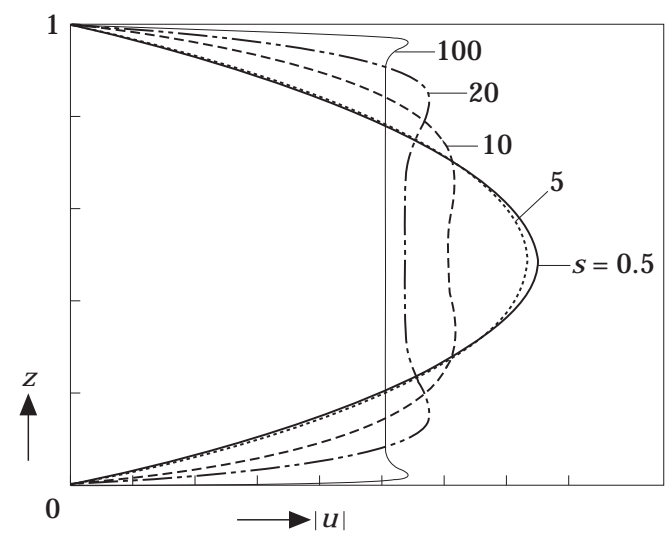

Figure 4. Velocity profile, magnitude. 


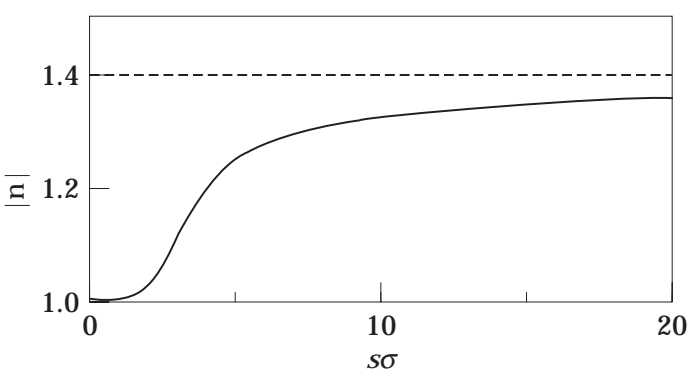

Figure 5. Magnitude of $n$.

system. When the pressure distribution in the air outside the gap is neglected, the forces acting on the plate are $\bar{F}_{e x}$, the harmonic excitation force generated by an electrodynamic shaker, $\bar{F}_{\text {gap }}$, the force acting on the lower side of the plate due to the pressure distribution in the gap between the plate and the fixed surface, and $\bar{F}_{\text {springs }}$, the spring forces; the plate is suspended on eight springs, two at each corner, and every spring has a stiffness $\kappa$.

The forces are written as

$$
\bar{F}_{e x}=l_{x} l_{y} p_{0} F_{e x} \mathrm{e}^{\mathrm{i} \omega t}, \quad \bar{F}_{g a p}=l_{x} l_{y} p_{0} F_{g a p} \mathrm{e}^{\mathrm{i} \omega t}, \quad \bar{F}_{\text {springs }}=8 \kappa h_{0} h \mathrm{e}^{\mathrm{i} \omega t} .
$$

From the equation of motion for the oscillating plate it follows that

$$
-\omega^{2} m h_{0} h=l_{x} l_{y} p_{0}\left[F_{e x}+F_{g a p}\right]-8 \kappa h_{0} h .
$$

The transfer function $H(\omega)$ relates the force and the displacement according to

$$
H(\omega)=h_{0} h / l_{x} l_{y} p_{0} F_{e x} .
$$

For a system with viscous damping it is more convenient to use the mobility function, representing the transfer function between velocity and force. The mobility function is obtained by multiplying $H(\omega)$ by $\mathrm{i} \omega$. Inserting the expressions for the air load, see (equation B.24), finally gives

$$
Y(\omega)=\mathrm{i} \omega /\left(-\omega^{2} m-\frac{l_{x} l_{y} p_{0}}{h_{0}}\left[\frac{32 k^{2} \gamma}{\pi^{2} g^{2} B(s)} \sum_{q=1,3,5 . .}^{\infty} \frac{1}{q^{2}} \frac{1}{D^{2}}\left[1-\frac{\tanh (D)}{D}\right]\right]+8 \kappa\right) .
$$

The eigenfrequency and the damping coefficient of the system are extracted from a Nyquist plot of the mobility function.

\subsection{PHYSICAL INTERPRETATION}

When the influence of the surrounding air is neglected and the structural damping is assumed to be negligible (this is confirmed in the tests described in section 7), the

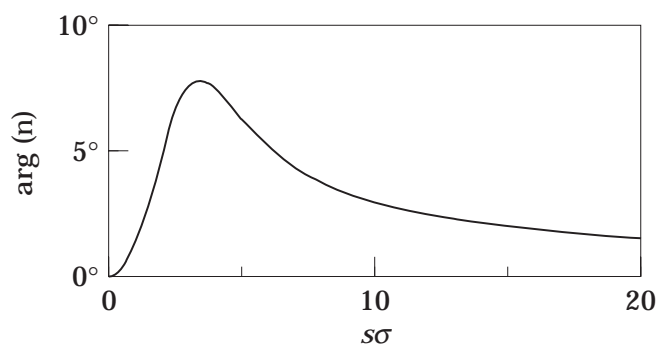

Figure 6. Phase angle of $n$. 
plate-spring system can be regarded as an undamped single-degree-of-freedom system with mass $m$ and stiffness $8 \kappa$. The surrounding air however will affect the dynamical behaviour of the plate. Usually the air load can be split up into an added mass, an added stiffness and an added damping. An analysis of the load term in the mobility function (28) however reveals that this concept is not straightforward for this situation. The expression for the resulting air load is a general, complex function of the angular frequency.

The imaginary part of the force could be used to extract the amount of added damping. Added mass and added stiffness however are, as in the experiments, not directly separable. In the calculations, however, one can define an artificial separation. For instance, the added stiffness can be related to the effects of compressibility. For this choice one obtains the amount of added mass by assuming the air to be incompressible. The added stiffness is then determined from the difference between compressible and incompressible behaviour.

However, the added stiffness would strongly depend on the frequency. This indicates that a split-up of the airload is rather arbitrary. Therefore the physical interpretation of the results will be based on a direct analysis of the force expression. Four aspects that influence the force are the narrowness of the gap, the viscosity, the aspect ratio and the compressibility.

\subsubsection{Narrowness of the gap}

One of the most important quantities that governs the air load is the narrowness of the gap, $g$. The force shows a strong increase with decreasing gap width. An analysis of the several terms concludes that for small gap widths the real part of the force is proportional to $h_{0}^{-1}$. The imaginary part, related to the damping, is proportional to $h_{0}^{-3}$ : it can be interpreted as a flow resistance term. In physical terms, the narrowness of the gap is associated with the amount of pumping in the gap. The motion of the air is mainly perpendicular to the motion of the plate (see Figure 7). It can easily be seen that this pumping effect increases with decreasing gap width.

\subsubsection{Aspect ratio of the plate}

The force is a function of the aspect ratio of the plate. It can easily be verified that for large aspect ratios the resulting force reduces to

$$
\bar{F}_{g a p}=\left(l_{x} l_{y} p_{0} / h_{0}\right)\left[4 k^{2} \gamma h / g^{2} B(s)\right] .
$$

\subsubsection{Viscosity}

A very interesting point is the role of the viscosity. The viscosity affects both the real and the imaginary part of the function $B(s)$. The damping depends on the viscosity, as expected. However, the eigenfrequency of the system is also affected by the viscosity. In this investigation the phase resonance frequency is used: the eigenfrequency of the corresponding undamped system.

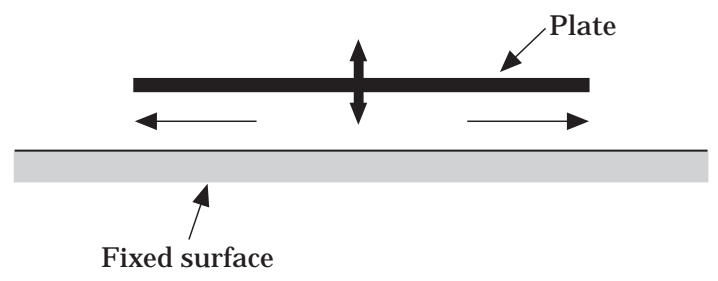

Figure 7. Pumping effect. 
Therefore the eigenfrequency is, by definition, not influenced by the damping. The eigenfrequency shift due to viscosity may seem surprising. Simply speaking one would expect the viscosity to affect the damping, but not the eigenfrequency. The change in frequency can be attributed to the change in the velocity profile in the gap. For low shear wave numbers there is a parabolic profile, whereas for high shear wave numbers the velocity profile is flat. It is therefore important that, even for a correct estimation of the eigenfrequency, the viscosity is taken into account.

\subsubsection{Compressibility}

In the calculations one can easily eliminate the effects of the compressibility. For incompressible behaviour, the function $D$ (see equation (22)), reduces to:

$$
D_{\text {inc }}=(q \pi / 2)(1 / a) .
$$

Compressibility effects are therefore important when:

$$
\left|k^{2} \gamma / g^{2} B(s) n\right| \cong(\pi / 2)^{2}(1 / a)^{2} .
$$

This can be rewritten as

$$
\left|\omega l_{y} / c_{e f f}(\omega)\right| \cong \pi / 2,
$$

where $c_{e f f}(\omega)$ is an effective speed of sound:

$$
c_{\text {eff }}(\omega)=c_{0} \sqrt{(n / \gamma) B(s)} .
$$

The effective speed of sound is affected by viscous effects, accounted for in $B(s)$, and thermal effects, accounted for in $n$. In physical terms, expression (32) now simply states that compressibility effects are important when the effective acoustic wave length is of the same order of magnitude as the plane length. This is in accordance with expectations.

\section{FINITE ELEMENT CALCULATIONS}

In order to compare the results from the analytical solution with the results from a standard technique, based on the wave equation, finite element calculations were carried out. In these calculations the air is represented as a compressible, inviscid medium. The finite element calculations were carried out for the experimental setup (see section 7) with the finite element program ANSYS 5.0. A rectangular solar panel is suspended on eight springs, two at each corner. The panel has a mass $m$ and (equivalent) Young's modulus $E$; each spring has a stiffness $\kappa$. The thickness of the panel is $0.022 \mathrm{~m}$. Because the plate is modelled as an elastic body, it will not be perfectly rigid. The deformation of the plate however is shown to be negligible in the frequency range of interest. This range is far below the first elastic eigenfrequency of the panel. The gap width between the plate and the fixed surface can be varied between 2 and $650 \mathrm{~mm}$. The following properties are taken according to the measurements:

$$
\begin{gathered}
m=2.516 \mathrm{~kg}, \quad \kappa=1178 \mathrm{~N} / \mathrm{m}, \quad E=2.61 \times 10^{9} \mathrm{~N} / \mathrm{m}^{2}, \\
l_{x}=0.49 \mathrm{~m}, \quad l_{y}=0.49 \mathrm{~m} .
\end{gathered}
$$

The properties of the surrounding air at standard atmospheric conditions are

$$
\rho_{0}=1 \cdot 2 \mathrm{~kg} / \mathrm{m}^{3}, \quad c_{0}=343 \mathrm{~m} / \mathrm{s} .
$$

The panel is modelled with three-dimensional eight-node solid elements, type SOLID45.

The degrees of freedom at each node are the displacements. In ANSYS the air is 


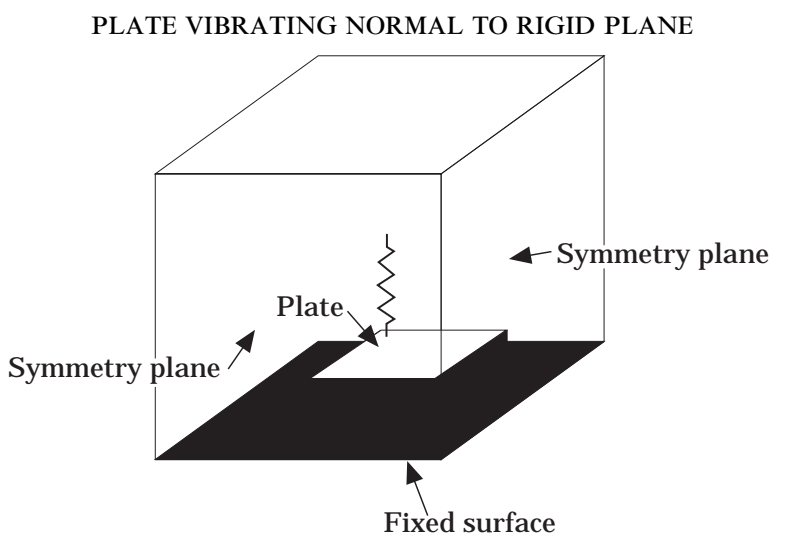

Figure 8. Finite element mesh.

represented as a compressible, inviscid medium. The behaviour of the air is therefore governed by the wave equation. Three-dimensional eight-node acoustic elements, type FLUID30, were used. For the acoustic elements, the pressure perturbation is the degree of freedom at each node.

With the use of symmetry the mesh is reduced to $1 / 4$ of the system (see Figure 8). In the numerical calculations, the two springs at each corner are replaced by a single spring with a double stiffness. The boundary condition for the planes of symmetry and the fixed surface is that the pressure gradient normal to the wall should vanish. In physical terms, this condition states that the velocity of the air normal to the surface is equal to zero. This condition is automatically satisfied in the ANSYS program when no other boundary conditions are specified.

The boundary conditions for the other air boundaries is chosen as $p=0$. This is a simplification of the Sommerfeld radiation condition, which states that all waves are outgoing. The condition $p=0$ however will give reliable results when the dimensions of the surrounding air domain are sufficiently large (without introducing standing waves of course). As a test case, the added mass was calculated for an oscillating plate in an infinite air domain for a number of mesh dimensions. The results learn that for an air domain of $1.50 \times 1.50$ (in-plane) $\times 2.85 \mathrm{~m}$ (perpendicular to the plate) for the $1 / 4$ system, the added mass differs only $3.8 \%$ from the value given in the literature [3]. This justifies the application of the boundary condition $p=0$ for the finite element calculations.

\section{COMPARISON WITH EXPERIMENTS}

\subsection{EXPERIMENTAL SETUP}

In order to measure the effects of the surrounding air, a relatively light and stiff plate has to be used. A solar panel is very well suited for this purpose. The measurements were carried out with a square ECS panel (by courtesy of Fokker Space \& Systems) of $0.98 \times 0.98 \mathrm{~m}$. The panel consists of two carbon skin plates separated by a honeycomb structure of thin aluminium sheet. The plate is suspended on eight springs, two located at each corner (see Figure 9). The panel is excited by an electrodynamic shaker, which is attached to the centre of the plate. The signal from an accelerometer is used as feedback for the shaker to keep the displacement amplitude constant. The fixed surface is a rectangular rigid plate of $2.20 \times 1.80 \mathrm{~m}$ which is mounted on the frame under the plate. Figure 9 shows the experimental set-up. The undamped eigenfrequency 
$f_{n}$ (phase resonance frequency) and the corresponding damping coefficient are measured as functions of the gap width. During the measurements the temperature is registered. It is used to exclude the influence of temperature differences on the density of the air. The influence of temperature on the other properties of the air is negligible. The properties of interest are

$$
\begin{gathered}
m=2.516 \mathrm{~kg}, \quad l_{x}=0.49 \mathrm{~m}, \quad l_{y}=0.49 \mathrm{~m}, \quad R_{0}=287 \mathrm{~J} / \mathrm{kgK}, \\
C_{p}=1004 \mathrm{~J} / \mathrm{kgK}, \quad C_{v}=716 \mathrm{~J} / \mathrm{kgK}, \\
\rho_{0}=1.2 \mathrm{~kg} / \mathrm{m}^{3}, \quad c_{0}=343 \mathrm{~m} / \mathrm{s}, \quad \lambda=25.6 \times 10^{-3} \mathrm{~W} / \mathrm{mK}, \\
\mu=18.2 \times 10^{-6} \mathrm{Ns} / \mathrm{m}^{2}, \quad k=1178 \mathrm{~N} / \mathrm{m}, \quad T_{0}=290 \mathrm{~K} .
\end{gathered}
$$

\subsection{DIMENSIONLESS PARAMETERS}

The experimental set-up is characterized by the value of the dimensionless parameters, as defined in section 3.1. The main parameter governing the motion of the air in the gap is the shear wave number. For large gaps the viscosity of the air can be neglected, whereas for small gaps viscous effects become dominant. The distance between the panel and the fixed surface, $h_{0}$, can be varied from 2 up to $650 \mathrm{~mm}$. For this range of gap widths the shear wave number varies between 1.28 and 1328. As $\sigma$ is a constant for air, the product $s \sigma$ varies between $0 \cdot 8$ and 1040 . This implies that for narrow gaps the process in the gap is isothermal, while for large gaps it occurs adiabatically.

These considerations indicate that even for a relatively large gap, e.g., $2 \mathrm{~mm}$, the shear wave number is very low. Because of the fact that the panel is very light, the amount of damping due to viscothermal effects will be substantial. In the literature most experiments are carried out at considerably higher frequencies. In order to obtain low shear wave numbers, one has to resort to very small gaps. Furthermore most experiments were carried

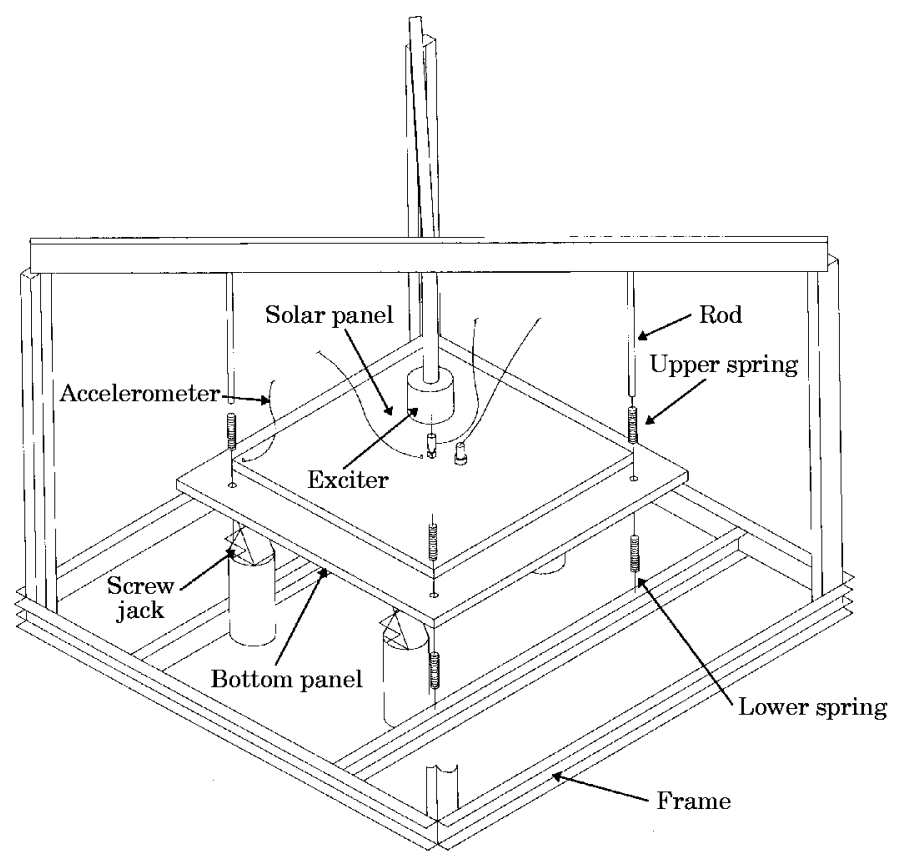

Figure 9. Experimental set-up. 
TABLE 2

Validation procedure, measurement results

\begin{tabular}{cccc}
\hline & \multicolumn{3}{c}{ Measured added mass $(\mathrm{kg})$} \\
\cline { 2 - 4 } Added mass $(\mathrm{kg})$ & $0 \cdot 0499$ & $h_{0}=35 \mathrm{~mm}$ & $h_{0}=10 \mathrm{~mm}$ \\
\hline $0 \cdot 0487$ & $0 \cdot 1042$ & $0 \cdot 0508$ & $0 \cdot 0506$ \\
$0 \cdot 1006$ & $0 \cdot 1493$ & $0 \cdot 1041$ & $0 \cdot 1026$ \\
$0 \cdot 1493$ & $0 \cdot 2056$ & $0 \cdot 1536$ & $0 \cdot 1541$ \\
$0 \cdot 2006$ & $0 \cdot 2040$ & $0 \cdot 2052$ \\
\hline
\end{tabular}

out with relatively stiff and heavy materials. The air loads will therefore have a relatively small influence.

It should be noted that by varying the gap width not only the value of the shear wave number is affected. Another important parameter, the reduced frequency $k$, varies between $10^{-5}$ and $0 \cdot 12$; the condition $k \ll 1$ is therefore satisfied. The geometrical parameter $g$ varies between 0.004 and 1.33 . This illustrates that the gap width can be varied from "very small" to "large".

\subsection{VALIDATION OF THE MEASUREMENT PROCEDURE}

The experimental set-up is used to measure the shift in eigenfrequency and the damping originating from the pressure distribution in the gap. The measurement procedure was validated by adding a known mass to the panel. The shift in phase resonance frequency was measured and used to determine the added mass. The measurement results for three different gap widths are listed in Table 2. The table shows that the added mass was measured to be within $3 \%$ of the actual, manually added, mass. So, it can be concluded that the set-up is suited for the measurements in this investigation.

\subsection{ACCURACY OF THE MEASUREMENTS}

The accuracy of the experiments is affected by several mechanisms. In order to determine the accuracy of the results, a number of tests were carried out.

\subsubsection{Parasitic oscillations}

Parasitic oscillations will affect the dynamical behaviour of the system. In order to determine if any parasitic motions were present, accelerations of the panel and the frame were measured in several directions. Two parasitic oscillations, an in-plane motion of the plate and a frame vibration, were identified. Structural modifications were applied whereby the parasitic motions were eliminated.

\subsubsection{Deformation of the panel}

Another oscillation that might affect the accuracy of the results is the deformation of the panel. The first elastic eigenfrequency of the panel in air is $49 \mathrm{~Hz}$. In order to consider the panel as rigid, the frequency range of interest should be far below $49 \mathrm{~Hz}$.

The stiffness of the springs was chosen in such a way that the first eigenfrequency of the plate-spring system in vacuum is $9.74 \mathrm{~Hz}$. Due to the influence of the surrounding air, the eigenfrequency will decrease compared to that for the system situation in vacuum. The frequency range of interest therefore was chosen to be $1-10 \mathrm{~Hz}$, and the panel was assumed to be rigid. During the experiments, the deformation of the plate was measured. With three accelerometers, mounted on the plate, the accelerations were measured to check if the plate 
indeed behaves rigidly. The measurements showed that the accelerations of the points mutually differ at most by $4 \%$, which justifies the assumption of rigidness.

\subsubsection{Compliance of the fixed surface}

The compliance of the fixed surface was measured with accelerometers The measurements showed that the amplitude of oscillation for the fixed surface is less than $1 \%$ of the amplitude of oscillation of the panel. The fixed surface can therefore be regarded as rigid.

\subsubsection{Tilting of the fixed surface}

In order to determine the influence of tilting, measurements were carried out for small tilting angles. For gap widths of 35, 10 and $6 \mathrm{~mm}$, tilting angles of respectively $1 \cdot 2,0.5$ and 0.5 degrees were used. For the panel of interest, with a length of $0.98 \mathrm{~m}$, this means a variation in gap width of respectively 20,2 and $2 \mathrm{~mm}$. The results show that the added mass and the added damping differ less than $5 \%$ for these tilting angles. Because of the fact that global quantities (eigenfrequency and damping) are measured, the sensitivity for small tilting angles is limited.

\subsubsection{Planeness of the surfaces}

The most important quantity that governs the accuracy of the experimental results is the planeness of the surfaces. Due to the shape of both the panel and the fixed surface the accuracy is affected. For a certain gap width the distance between the panel and the fixed surface was measured at several points between the panel and the fixed surface. The gap width was not constant but varied with position: $\pm 0.8 \mathrm{~mm}$. This could mainly be attributed to the non-planeness of the fixed surface. With a least squares technique the accuracy was improved. The gap width data were used to find the position of the fixed surface that would lead to the best constant gap width. Correspondingly the gap width was then adjusted in three points for each measurement. Due to this correction, the error which is introduced by the non-planeness of the surfaces could be reduced to $\pm 0.4 \mathrm{~mm}$ in gap width. The measurements could therefore be carried out with a satisfying accuracy, especially in respect to the accuracies reported in previous papers in this area.

\subsubsection{Non-linear effects}

The theory used in the present investigation is based on linearized equations. In the experiments the amplitude of oscillation of the panel therefore has to be small compared to the gap width. In order to determine if any non-linear effects are present a number of

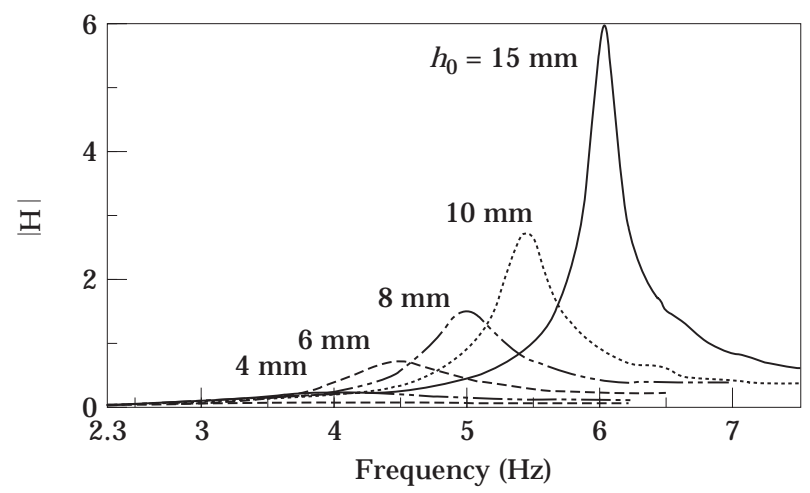

Figure 10. Amplitude of transfer function $H(\omega)$. 
PLATE VIBRATING NORMAL TO RIGID PLANE

TABLE 3

Experimental results

\begin{tabular}{cccccc}
\hline$h_{0}(\mathrm{~mm})$ & $f_{n}(\mathrm{~Hz})$ & $\xi(\%)$ & $s$ & $k$ & $g$ \\
\hline 650 & $8 \cdot 557$ & $0 \cdot 20$ & 1223 & $10 \cdot 3 \mathrm{E}-02$ & $1 \cdot 33 \mathrm{E}+00$ \\
150 & $8 \cdot 303$ & $0 \cdot 12$ & 278 & $23 \cdot 0 \mathrm{E}-03$ & $30 \cdot 6 \mathrm{E}-02$ \\
80 & $7 \cdot 994$ & $0 \cdot 20$ & 146 & $11 \cdot 8 \mathrm{E}-03$ & $16 \cdot 3 \mathrm{E}-02$ \\
50 & $7 \cdot 635$ & $0 \cdot 32$ & $88 \cdot 9$ & $7 \cdot 05 \mathrm{E}-03$ & $10 \cdot 2 \mathrm{E}-02$ \\
35 & $7 \cdot 277$ & $0 \cdot 52$ & $60 \cdot 8$ & $4 \cdot 71 \mathrm{E}-03$ & $71 \cdot 4 \mathrm{E}-03$ \\
25 & $6 \cdot 871$ & $0 \cdot 84$ & $42 \cdot 2$ & $3 \cdot 14 \mathrm{E}-03$ & $51 \cdot 0 \mathrm{E}-03$ \\
15 & $6 \cdot 139$ & $1 \cdot 83$ & $23 \cdot 9$ & $1 \cdot 70 \mathrm{E}-03$ & $30 \cdot 6 \mathrm{E}-03$ \\
12 & $5 \cdot 767$ & $2 \cdot 60$ & $18 \cdot 5$ & $1 \cdot 28 \mathrm{E}-03$ & $24 \cdot 5 \mathrm{E}-03$ \\
10 & $5 \cdot 441$ & $3 \cdot 51$ & $15 \cdot 0$ & $1 \cdot 01 \mathrm{E}-04$ & $20 \cdot 4 \mathrm{E}-03$ \\
8 & $5 \cdot 050$ & $5 \cdot 04$ & $11 \cdot 6$ & $7 \cdot 47 \mathrm{E}-04$ & $16 \cdot 3 \mathrm{E}-03$ \\
6 & $4 \cdot 533$ & $8 \cdot 24$ & $8 \cdot 22$ & $5 \cdot 03 \mathrm{E}-04$ & $12 \cdot 2 \mathrm{E}-03$ \\
5 & $4 \cdot 217$ & $11 \cdot 59$ & $6 \cdot 61$ & $3 \cdot 90 \mathrm{E}-04$ & $10 \cdot 2 \mathrm{E}-03$ \\
4 & $3 \cdot 839$ & $18 \cdot 78$ & $5 \cdot 04$ & $2 \cdot 84 \mathrm{E}-04$ & $8 \cdot 16 \mathrm{E}-03$ \\
$3 \cdot 5$ & $3 \cdot 599$ & $24 \cdot 55$ & $4 \cdot 27$ & $2 \cdot 33 \mathrm{E}-04$ & $7 \cdot 14 \mathrm{E}-03$ \\
3 & $3 \cdot 337$ & $35 \cdot 71$ & $3 \cdot 53$ & $1 \cdot 85 \mathrm{E}-04$ & $6 \cdot 12 \mathrm{E}-03$ \\
\hline
\end{tabular}

experiments were carried out with different amplitudes of oscillation. For gap widths of 50,15 and $8 \mathrm{~mm}$ measurements were performed with amplitudes varying from 0.3 up to $1.8 \mathrm{~mm}$. In all situations the eigenfrequency and the damping were not affected by the amplitude of motion.

\subsubsection{Flow around plate edges}

The flow around the plate edges was visualized with smoke tests. In the frequency and amplitude range of interest the motion of the air was laminar. No vortices or irregularies in the flow pattern were observed.

\subsection{EXPERIMENTAL RESULTS}

Typical results for the transfer function are given in Figure 10. This figure learns that the shift in eigenfrequency is relatively large. If one assigns this frequency shift to an added mass effects, this reveals that the added mass of the air is relatively large. The strong increase in added mass is illustrated by the following example: for a gap of $3 \mathrm{~mm}$, the amount of added mass for the experimental set-up would be about $22 \mathrm{~kg}$, while the mass of the panel is only $2.5 \mathrm{~kg}$ ! The sharp increase in damping is illustrated by the flattening

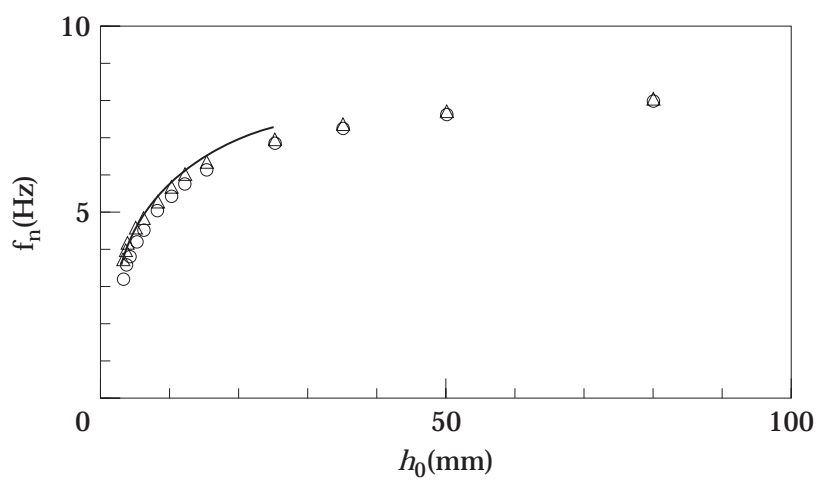

Figure 11. Eigenfrequency versus gap width. $\triangle$, ANSYS 5.0; $\bigcirc$, experiment; - , analytical. 


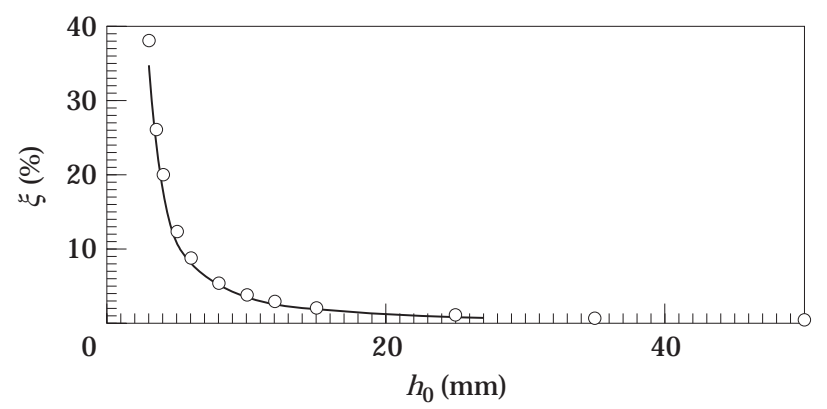

Figure 12. Damping coefficient versus gap width. $\bigcirc$, Experiment; — , analytical.

of the transfer function. The pumping effect induces high viscous losses, which results in damping of the panel. For a gap of $3 \mathrm{~mm}$, the dimensionless damping coefficient $\xi$ is $35 \%$ : the panel is almost critically damped. The experimental results are summarized in Table 3. For each gap width the eigenfrequency, the damping coefficient and the dimensionless parameters are listed.

\subsection{COMPARISON BETWEEN THEORY AND EXPERIMENTS}

The measured and the calculated eigenfrequencies are depicted in Figure 11. Because of the fact that, due to a change in gap width $h_{0}$, a number of dimensionless parameters change in value, the figure is not plotted in a non-dimensional form. Figure 11 shows that there is a strong decrease in eigenfrequency with decreasing gap width. The results from both the finite element calculations and the "narrow gap" solution show fair agreement with the experimental results. For very small gaps the finite element calculations overestimate the eigenfrequency because the viscosity of the air is neglected.

The corresponding dimensionless damping coefficients are depicted in Figure 12. This figure shows that there is a strong increase in damping with decreasing gap width. The results from the "narrow gap" solution show good agreement with the experimental results. It is evident that the damping coefficients for the finite element calculations are zero, because the viscosity of the air is neglected.

\section{CONCLUSIONS}

The conclusions to be drawn from the present investigation are as follows.

For narrow gaps an analytical solution was obtained. The "narrow gap" solution includes the effects of inertia, viscosity, compressibility and thermal conductivity.

The motion of a rigid plate oscillating normal to a fixed surface is governed by a number of dimensionless parameters: the shear wave number, the reduced frequency, the ratio of specific heats, the square root of the Prandtl number, the narrowness of the gap and the aspect ratio of the plate. With these dimensionless parameters the range of validity can, in contrast with previous publications in this area, be determined relatively easy.

The shear wave number, which is a measure for the ratio between inertial forces and viscous forces, indicates that for narrow gaps the viscosity of the air has to be taken into account.

A special experimental set-up was designed with the use of the dimensionless parameters. Substantial frequency shifts and very large damping values were measured with satisfying accuracy.

Calculations and experiments show fair agreement; the transition from inertial dominated flow to viscous dominated flow is demonstrated. The viscosity of the air causes 
a strong damping effect for narrow gaps, which is an interesting point regarding the practical application of this research.

\section{ACKNOWLEDGMENTS}

The authors owe many thanks to Bert Wolbert, under whose supervision the measurements have been performed, and Egbert van de Veen and Andre Glas, who carried out the experiments. The support from SHELL and Fokker Space is gratefully acknowledged.

\section{REFERENCES}

\section{General}

1. W. M. Beltman 1994 University of Twente report WB.94/TM-943. Forces on a rigid plate oscillating normal to a fixed surface.

2. H. TiJdeman 1975 Journal of Sound and Vibration 39, 1-33. On the propagation of sound waves in cylindrical tubes.

3. W. K. Meyerhoff 1970 Journal of Ship Research 14, 100-111. Added mass of thin rectangular plates calculated from potential theory.

\section{Fluid mechanics}

4. H. Lamb 1932 Hydrodynamics (6th edition). New York: Dover.

5. E. C. Kuhn and C. C. Yates 1964 ASLE Transactions 7, 299-303. Fluid inertia effect on the film pressure between axially oscillating parallel circular plates.

6. R. M. TERriLl 1969 Journal of Lubrication Technology 91, 126-131. The flow between two parallel circular disks, one of which is subject to a normal sinusoidal oscillation.

7. S. Ishizawa 1966 Japanese Society of Mechanical Engineering 9.

8. C. Y. Wang and B. Drachman 1982 Applied Scientific Research 39, 55-68. The steady streaming generated by a vibrating plate parallel to a fixed plate.

9. V. Ramamurthy and U. S. Rao 1987 Fluid Dynamics Research 2, 47-63. The steady streaming generated by a vibrating plate parallel to a fixed plate in a dusty fluid.

10. J. Stefan 1874 Math. Naturwissenschaftliche Klasse, Wien. Sitzungsberichte 69, 713. Versuche über die scheinbare Adhesion.

11. O. Reynolds 1886 Philosophical Transactions of the Royal Society of London Series A 177, 157-234. On the theory of lubrication.

12. W. Kahlert 1948 Ingenieur Arch. 16, 321-342. Der Einfluß der Trägheitskräfte bei der hydrodynamischen Schmiermittel theorie (in German).

13. R. S. Brand 1955 American Society of Mechanical Engineers Transactions 77, 363-364. Inertia forces in lubricating films.

14. W. S. Griffin, H. H. Richardson and S. Yamanami 1966 Journal of Basic Engineering 88 451-456. A study of fluid squeeze film damping.

15. J. V. Beck, W. G. Holliday and G. L. Strodtman 1969 Journal of Lubrication Technology 91 138-148. Experiment and analysis of a flat disk squeeze-film bearing including effects of supported mass motion.

16. J. A. Tichy and M. F. Modest 1978 Journal of Lubrication Technology 100, 316-222. Squeeze film flow between arbitrary two-dimensional surfaces subject to normal oscillations.

17. J. Prakash and H. Christensen 1978 Journal of Mechanical Engineering Science 20, 183-188. Squeeze film between two rough rectangular plates.

18. S. M. R. Hashemi and B. J. Roylance 1989 Tribology Transactions 32, 461-468. Analysis of an oscillatory oil squeeze film including effects of fluid inertia.

Acoustics

19. E. E. Ungar and J. R. Carbonell 1966 American Institute of Aeronautics and Astronautics Journal 4, 1385-1390. On panel vibration damping due to structural joints.

20. G. MaIdANiK 1966 Journal of the Acoustical Society of America 40, 1064-1072. Energy dissipation associated with gas-pumping in structural joints.

21. M. J. H. Fox and P. N. WhitTon 1980 Journal of Sound and Vibration 73, 279-295. The damping of structural vibrations by thin gas films.

22. A. Trochidis 1982 Acustica 51, 201-212. Vibration damping due to air or liquid layers. 
23. A. MösER 1980 Acustica 46, 210-217. Damping of structure-borne sound by the viscosity of a layer between two plates.

24. L. C. Chow and R. J. Pinnington 1986 Journal of Sound and Vibration 118, 123-139. Practical industrial methods of increasing structural damping in machinery, I: squeeze film damping with air.

25. L. C. Chow and R. J. Pinnington 1988 Journal of Sound and Vibration 128, 333-347. Practical industrial methods of increasing structural damping in machinery, II: squeeze film damping with liquids.

26. T. Önsay 1993 Journal of Sound and Vibration 163, 231-259. Effects of layer thickness on the vibration response of a plate-fluid layer system.

27. C. A. TAN and C. D. Mote JR. 1991 International Journal of Engineering Science 29, 1609-1625. Pressure and flow in fluid films constrained between translating and vibrating flexible surfaces.

28. T. T. YEH and S. S. CHEN 1978 Journal of Sound and Vibration 59, 453-467. The effect of fluid viscosity on coupled tube/fluid vibrations.

29. M. Bruneau, A. M. Bruneau and P. Hamery 1993 Acustica with Acta Acustica 1, 227-234. An improved approach to modeling the behaviour of thin fluid films trapped between a vibrating membrane and a backing wall and surrounded by a reservoir at the periphery.

\section{APPENDIX A: BASIC EQUATIONS}

The equations governing the motion of the air between an oscillating plate parallel to a fixed surface are as follows: (a) the Navier-Stokes equations, for a constant value of the viscosity, $\mu$,

$$
\begin{aligned}
& \bar{\rho}\left[\frac{\partial \bar{u}}{\partial t}+\bar{u} \frac{\partial \bar{u}}{\partial \bar{x}}+\bar{v} \frac{\partial \bar{u}}{\partial \bar{y}}+\bar{w} \frac{\partial \bar{u}}{\partial \bar{z}}\right]=-\frac{\partial \bar{p}}{\partial \bar{x}}+\mu\left[\frac{\partial^{2} \bar{u}}{\partial \bar{x}^{2}}+\frac{\partial^{2} \bar{u}}{\partial \bar{y}^{2}}+\frac{\partial^{2} \bar{u}}{\partial \bar{z}^{2}}\right]+\frac{1}{3} \mu \frac{\partial}{\partial \bar{x}}\left[\frac{\partial \bar{u}}{\partial \bar{x}}+\frac{\partial \bar{v}}{\partial \bar{y}}+\frac{\partial \bar{w}}{\partial \bar{z}}\right], \\
& \bar{\rho}\left[\frac{\partial \bar{v}}{\partial t}+\bar{u} \frac{\partial \bar{v}}{\partial \bar{x}}+\bar{v} \frac{\partial \bar{v}}{\partial \bar{y}}+\bar{w} \frac{\partial \bar{v}}{\partial \bar{z}}\right]=-\frac{\partial \bar{p}}{\partial \bar{y}}+\mu\left[\frac{\partial^{2} \bar{v}}{\partial \bar{x}^{2}}+\frac{\partial^{2} \bar{v}}{\partial \bar{y}^{2}}+\frac{\partial^{2} \bar{v}}{\partial \bar{z}^{2}}\right]+\frac{1}{3} \mu \frac{\partial}{\partial \bar{y}}\left[\frac{\partial \bar{u}}{\partial \bar{x}}+\frac{\partial \bar{v}}{\partial \bar{y}}+\frac{\partial \bar{w}}{\partial \bar{z}}\right], \\
& \bar{\rho}\left[\frac{\partial \bar{w}}{\partial t}+\bar{u} \frac{\partial \bar{w}}{\partial \bar{x}}+\bar{v} \frac{\partial \bar{w}}{\partial \bar{y}}+\bar{w} \frac{\partial \bar{w}}{\partial \bar{z}}\right]=-\frac{\partial \bar{p}}{\partial \bar{z}}+\mu\left[\frac{\partial^{2} \bar{w}}{\partial \bar{x}^{2}}+\frac{\partial^{2} \bar{w}}{\partial \bar{y}^{2}}+\frac{\partial^{2} \bar{w}}{\partial \bar{z}^{2}}\right]+\frac{1}{3} \mu \frac{\partial}{\partial \bar{z}}\left[\frac{\partial \bar{u}}{\partial \bar{x}}+\frac{\partial \bar{v}}{\partial \bar{y}}+\frac{\partial \bar{w}}{\partial \bar{z}}\right] ;
\end{aligned}
$$

(b) the equation of continuity,

$$
\frac{\partial \bar{\rho}}{\partial t}+\frac{\partial(\bar{\rho} \bar{u})}{\partial \bar{x}}+\frac{\partial(\bar{\rho} \bar{v})}{\partial \bar{y}}+\frac{\partial(\bar{\rho} \bar{w})}{\partial \bar{z}}=0
$$

(c) the equation of state for an ideal gas,

$$
\bar{p}=\bar{\rho} R_{0} \bar{T},
$$

where $R_{0}$ can be written as:

$$
R_{0}=p_{0} / \rho_{0} T_{0} ;
$$

(d) the energy equation, for a constant value of the thermal conductivity $\lambda$ and no internal heat generation, 


$$
\begin{aligned}
\bar{\rho} C_{p}\left[\frac{\partial \bar{T}}{\partial t}+\bar{u} \frac{\partial \bar{T}}{\partial \bar{x}}+\bar{v} \frac{\partial \bar{T}}{\partial \bar{y}}+\bar{w} \frac{\partial \bar{T}}{\partial \bar{z}}\right]=\lambda\left[\frac{\partial^{2} \bar{T}}{\partial \bar{x}^{2}}\right. & \left.+\frac{\partial^{2} \bar{T}}{\partial \bar{y}^{2}}+\frac{\partial^{2} \bar{T}}{\partial \bar{z}^{2}}\right] \\
& +\frac{\partial \bar{p}}{\partial t}+\bar{u} \frac{\partial \bar{p}}{\partial \bar{x}}+\bar{v} \frac{\partial \bar{p}}{\partial \bar{y}}+\bar{w} \frac{\partial \bar{p}}{\partial \bar{z}}+\mu \Phi,
\end{aligned}
$$

where $\Phi$ is the viscous dissipation function

$$
\begin{aligned}
\Phi= & 2\left[\left(\frac{\partial \bar{u}}{\partial \bar{x}}\right)^{2}+\left(\frac{\partial \bar{v}}{\partial \bar{y}}\right)^{2}+\left(\frac{\partial \bar{w}}{\partial \bar{z}}\right)^{2}\right]-\frac{2}{3}\left[\frac{\partial \bar{u}}{\partial \bar{x}}+\frac{\partial \bar{v}}{\partial \bar{y}}+\frac{\partial \bar{w}}{\partial \bar{z}}\right]^{2}+\left[\frac{\partial \bar{u}}{\partial \bar{y}}+\frac{\partial \bar{v}}{\partial \bar{x}}\right]^{2} \\
& +\left[\frac{\partial \bar{v}}{\partial \bar{z}}+\frac{\partial \bar{w}}{\partial \bar{y}}\right]^{2}+\left[\frac{\partial \bar{w}}{\partial \bar{x}}+\frac{\partial \bar{u}}{\partial \bar{z}}\right]^{2} .
\end{aligned}
$$

Assuming sinusoidal motion and no mean flow, one has

$$
\begin{aligned}
\bar{u}=c_{0} u(\bar{x}, \bar{y}, \bar{z}) \mathrm{e}^{\mathrm{i} \omega t}, & \bar{p}=p_{0}\left[1+p(\bar{x}, \bar{y}, \bar{z}) \mathrm{e}^{\mathrm{i} \omega t}\right], \quad \bar{v}=c_{0} v(\bar{x}, \bar{y}, \bar{z}) \mathrm{e}^{\mathrm{i} \omega t}, \\
\bar{p}=\rho_{0}\left[1+\rho(\bar{x}, \bar{y}, \bar{z}) \mathrm{e}^{\mathrm{i} \omega t}\right], & \bar{w}=c_{0} w(\bar{x}, \bar{y}, \bar{z}) \mathrm{e}^{\mathrm{i} \omega t}, \quad \bar{T}=T_{0}\left[1+T(\bar{x}, \bar{y}, \bar{z}) \mathrm{e}^{\mathrm{i} \omega t}\right],
\end{aligned}
$$

with $u, v, w, p, \rho$ and $T$ being small dimensionless perturbations. The mean pressure and the mean density are related according to $p_{0}=\rho_{0} c_{0}^{2} / \gamma$.

Dimensionless co-ordinates are introduced:

$$
x=\bar{x} / l_{x}, \quad y=\bar{y} / l_{y}, \quad z=\bar{z} / h_{0} .
$$

When higher order terms are neglected, the equation can be written in a linearized, dimensionless form. The equations are

$$
\begin{gathered}
\mathrm{i} u=-\frac{g}{k} \frac{1}{\gamma} \frac{\partial p}{\partial x}+\frac{1}{s^{2}}\left[g^{2} \frac{\partial^{2} u}{\partial x^{2}}+\left(\frac{g}{a}\right)^{2} \frac{\partial^{2} u}{\partial y^{2}}+\frac{\partial^{2} u}{\partial z^{2}}\right]+\frac{1}{3} \frac{g}{s^{2}} \frac{\partial}{\partial x}\left[g \frac{\partial u}{\partial x}+\left(\frac{g}{a}\right) \frac{\partial v}{\partial y}+\frac{\partial w}{\partial z}\right] \\
\mathrm{i} v=-\frac{g}{a k} \frac{1}{\gamma} \frac{\partial p}{\partial y}+\frac{1}{s^{2}}\left[g^{2} \frac{\partial^{2} v}{\partial x^{2}}+\left(\frac{g}{a}\right)^{2} \frac{\partial^{2} v}{\partial y^{2}}+\frac{\partial^{2} v}{\partial z^{2}}\right]+\frac{1}{3} \frac{g}{a s^{2}} \frac{\partial}{\partial y}\left[g \frac{\partial u}{\partial x}+\left(\frac{g}{a}\right) \frac{\partial v}{\partial y}+\frac{\partial w}{\partial z}\right] \\
\mathrm{i} w=-\frac{1}{k} \frac{1}{\gamma} \frac{\partial p}{\partial z}+\frac{1}{s^{2}}\left[g^{2} \frac{\partial^{2} w}{\partial x^{2}}+\left(\frac{g}{a}\right)^{2} \frac{\partial^{2} w}{\partial y^{2}}+\frac{\partial^{2} w}{\partial z^{2}}\right]+\frac{1}{3} \frac{1}{s^{2}} \frac{\partial}{\partial z}\left[g \frac{\partial u}{\partial x}+\left(\frac{g}{a}\right) \frac{\partial v}{\partial y}+\frac{\partial w}{\partial z}\right] \\
g \frac{\partial u}{\partial x}+\left(\frac{g}{a}\right) \frac{\partial v}{\partial y}+\frac{\partial w}{\partial z}=-\mathrm{i} k \rho, \quad p=\rho+T \\
\mathrm{i} T=\frac{1}{s^{2} \sigma^{2}}\left[g^{2} \frac{\partial^{2} T}{\partial x^{2}}+\left(\frac{g}{a}\right)^{2} \frac{\partial^{2} T}{\partial y^{2}}+\frac{\partial^{2} T}{\partial z^{2}}\right]+\mathrm{i}\left[\frac{\gamma-1}{\gamma}\right] p
\end{gathered}
$$

where

$s=h_{0} \sqrt{\rho_{0} \omega / \mu}$ shear wave number, $\quad \sigma=\sqrt{\mu C_{p} / \lambda}$ square root of the Prandtl number,

$$
k=\omega h_{0} / c_{0} \text { reduced frequency, }
$$

$\gamma=C_{p} / C_{v}$ ratio of specific heats, $g=h_{0} / l_{x}$ narrowness of the gap,

$$
a=l_{y} / l_{x} \text { aspect ratio of the plate. }
$$




\section{APPENDIX B: "NARROW GAP” SOLUTION}

When the gap width is small in comparison with the dimensions of the plate, and the velocity $w$ is negligible with respect to the in-plane velocities $u$ and $v$ (i.e. $g \ll 1, g / s \ll 1$, $w / v \ll 1$ and $w / u \ll 1)$, the equations (A11-16) can be simplified to

$$
\begin{gathered}
\mathrm{i} u=-\frac{g}{k} \frac{1}{\gamma} \frac{\partial p}{\partial x}+\frac{1}{s^{2}} \frac{\partial^{2} u}{\partial z^{2}}, \quad \mathrm{i} v=-\frac{g}{a k} \frac{1}{\gamma} \frac{\partial p}{\partial y}+\frac{1}{s^{2}} \frac{\partial^{2} v}{\partial z^{2}}, \quad 0=-\frac{1}{k} \frac{1}{\gamma} \frac{\partial p}{\partial z}, \\
g \frac{\partial u}{\partial x}+\left(\frac{g}{a}\right) \frac{\partial v}{\partial y}+\frac{\partial w}{\partial z}=-\mathrm{i} k \rho, \quad p=\rho+T, \quad \mathrm{i} T=\frac{1}{s^{2} \sigma^{2}} \frac{\partial^{2} T}{\partial z^{2}}+\mathrm{i}\left[\frac{\gamma-1}{\gamma}\right] p .
\end{gathered}
$$

The boundary conditions are as follows:

$$
\begin{gathered}
\text { at } z=0, \quad u=0, \quad v=0, \quad w=0, \quad T=0 \text { (isothermal wall); } \quad \text { at } z=1 \\
u=0, \quad v=0, \quad w=\mathrm{i} k h, \quad T=0 \text { (isothermal wall); at } x=-1 \quad p=0, \\
\text { at } x=1 \quad p=0 ; \quad \text { at } y=-1 \quad p=0, \quad \text { at } y=1 \quad p=0 . \quad \text { (B } 7)
\end{gathered}
$$

From equation (B3) it follows that the magnitude of the pressure perturbation, $p$, depends only on the in-plane coordinates $x$ and $y$. Because the pressure perturbation is independent of the co-ordinate $z$, the solution for equation (B6) can be written as:

$$
T=[(\gamma-1) / \gamma] p A(s \sigma, z),
$$

where

$$
A(s \sigma, z)=[(\sinh (s \sigma \sqrt{\mathrm{i}}(z-1))-\sinh (s \sigma \sqrt{\mathrm{i} z})) / \sinh (s \sigma \sqrt{\mathrm{i}})+1] .
$$

Substitution of equation (B8) into equation (B5) gives

$$
\rho=p\{1-[(\gamma-1) / \gamma] A(s \sigma, z)\} .
$$

The solution for equation (B1) can be written in the form

$$
u=\mathrm{i}(g / k)(1 / \gamma)(\partial p / \partial x) A(s, z) .
$$

Similarly, it follows that

$$
v=\mathrm{i}(g / a k)(1 / \gamma)(\partial p / \partial y) A(s, z) .
$$

Finally, the equation of continuity (B4) has to be satisfied. Inserting the equations (B11), (B12) and (B10) gives

$$
\mathrm{i} \frac{g^{2}}{k} \frac{1}{\gamma}\left(\frac{\partial^{2} p}{\partial x^{2}}\right) A(s, z)+\mathrm{i} \frac{g^{2}}{a^{2} k} \frac{1}{\gamma}\left(\frac{\partial^{2} p}{\partial y^{2}}\right) A(s, z)+\frac{\partial w}{\partial z}=-\mathrm{i} k p\left\{1-\left[\frac{\gamma-1}{\gamma}\right] A(s \sigma, z)\right\} .
$$

Integrating with respect to $z$ gives

$$
\mathrm{i} \frac{g^{2}}{k} \frac{1}{\gamma}\left(\frac{\partial^{2} p}{\partial x^{2}}\right) B(s)+\mathrm{i} \frac{g_{y}^{2}}{a^{2} k} \frac{1}{\gamma}\left(\frac{\partial^{2} p}{\partial y^{2}}\right) B(s)+w_{1}-w_{0}=-\mathrm{i} k p\left\{1-\left[\frac{\gamma-1}{\gamma}\right] B(s \sigma)\right\} .
$$

where

$$
B(s) \equiv \int_{z=0}^{1} A(s, z) \mathrm{d} z=2\left[\frac{1-\cosh (s \sqrt{\mathrm{i}})}{s \sqrt{\mathrm{i}} \sinh (s \sqrt{\mathrm{i}})}\right]+1
$$


Rearranging equation (B14) for the pressure perturbation $p$ yields

$$
g^{2}\left(\frac{\partial^{2} p}{\partial x^{2}}\right)+\left(\frac{g}{a}\right)^{2}\left(\frac{\partial^{2} p}{\partial y^{2}}\right)=\frac{k \gamma}{B(s)}\left[-k p\left\{1-\left[\frac{\gamma-1}{\gamma}\right] B(s \sigma)\right\}+\mathrm{i}\left\{w_{1}-w_{0}\right\}\right] .
$$

The dimensionless velocities $w_{1}$ and $w_{0}$ can be expressed as in equations (B7). Substitution of equations (B7) into equation (B16) finally gives the equation for the pressure perturbation $p$ in the gap:

$$
g^{2}\left(\frac{\partial^{2} p}{\partial x^{2}}\right)+\left(\frac{g}{a}\right)^{2}\left(\frac{\partial^{2} p}{\partial y^{2}}\right)=-\frac{k^{2} \gamma}{B(s)}\left[p\left\{1-\left[\frac{\gamma-1}{\gamma}\right] B(s \sigma)\right\}+h\right] .
$$

From equation (B17) the expression for the (complex) polytropic constant $n$ can be obtained (note that this constant was obtained by integrating over the layer thickness):

$$
n=[1-[(\gamma-1) / \gamma] B(s \sigma)]^{-1} .
$$

Substitution of expression (B18) in equation (B17) gives the "narrow gap" equation:

$$
g^{2}\left(\frac{\partial^{2} p}{\partial x^{2}}\right)+\left(\frac{g}{a}\right)^{2}\left(\frac{\partial^{2} p}{\partial y^{2}}\right)=-\frac{k^{2} \gamma}{B(s)}\left[\frac{p}{n}+h\right] .
$$

Equation (B19) is solved by assuming

$$
p=\sum_{q=1,3,5 .}^{\infty} M(x) \cos \left(\frac{q \pi y}{2}\right) .
$$

By this assumption the boundary conditions for $p$ at $y=-1$ and $y=1$ are automatically satisfied. Substitution of expression (B20) in equation (B19) gives a differential equation for the function $M$. Solving for $M$ from this equation and taking into account the boundary conditions gives

$$
M=\frac{4}{\pi g^{2} q} \frac{k^{2} \gamma h}{B(s)} \frac{(-1)^{(q-1) / 2}}{\left[\left(\frac{q \pi}{2}\right)^{2}\left(\frac{1}{a}\right)^{2}-\frac{k^{2} \gamma}{g^{2} B(s) n}\right]}\left[1-\frac{\cosh \left(\sqrt{\left(\frac{q \pi}{2}\right)^{2}\left(\frac{1}{a}\right)^{2}-\frac{k^{2} \gamma}{g^{2} B(s) n} x}\right)}{\cosh \left(\sqrt{\left(\frac{q \pi}{2}\right)^{2}\left(\frac{1}{a}\right)^{2}-\frac{k^{2} \gamma}{g^{2} B(s) n}}\right)}\right] .
$$

The function $\mathrm{D}$ is introduced according to

$$
D=\sqrt{(q \pi / 2)^{2}(1 / a)^{2}-k^{2} \gamma / g^{2} B(s) n} .
$$

Finally the solution for the pressure perturbation is obtained:

$$
p=\frac{4}{\pi} \frac{1}{g^{2}} \frac{k^{2} \gamma h}{B(s)} \sum_{q=1,3,5 .}^{\infty} \frac{1}{q} \frac{(-1)^{(q-1) / 2}}{D^{2}}\left[1-\frac{\cosh (D x)}{\cosh (D)}\right] \cos \left(\frac{q \pi}{2} y\right)
$$

The dimensionless force, acting on the lower side of the plate, can be obtained by 
integration. This gives

$$
F_{g a p}=\frac{32}{\pi^{2}} \frac{1}{g^{2}} \frac{k^{2} \gamma h}{B(s)} \sum_{q=1,3,5 . .}^{\infty} \frac{1}{q^{2}} \frac{1}{D^{2}}\left[1-\frac{\tanh (D)}{D}\right] .
$$

Now that the pressure distribution is known, all quantities can be obtained. This is done by inserting the solution for $p$ in the appropriate equations. The temperature perturbation $T$ can be obtained from equation (B8). Equation (B10) gives the density distribution. Equations (B11) and (B12) are used to obtain the velocity perturbations $u$ and $v$ respectively. The velocity $w$ can be calculated by integrating equation (B4) with respect to $z$.

\section{APPENDIX C: BEHAVIOUR OF THE NARROW GAP SOLUTION FOR LOW SHEAR WAVE NUMBERS: REYNOLDS EQUATION}

For low shear wave numbers the inertial forces can be neglected compared to the viscous forces. In this situation the function $B(s)$ (see section 4.1 and Appendix B) can be approximated by

$$
B(s) \cong \frac{1}{12} \mathrm{i} s^{2}
$$

The polytropic constant $n$ reduces to 1 for low shear wave numbers. This means that the process occurs isothermally for low shear wave numbers. A low shear wave number implies that inertial effects can be neglected. The "narrow gap" equation for the pressure perturbation $p$ (equation (B19)) now reduces to

$$
g^{2} \partial^{2} p / \partial x^{2}+(g / a)^{2} \partial^{2} p / \partial y^{2}-\left(12 \mathrm{i} k^{2} \gamma / s^{2}\right) p=\left(12 \mathrm{i} k^{2} \gamma / s^{2}\right) h
$$

This equation is a modified form of the Reynolds equation. Equation $(\mathrm{C} 2)$ can be derived directly from the complete, non-linear Reynolds equation. In this case no stretch or wedge effects are present. When small perturbations and dimensionless co-ordinates are introduced, equation $(\mathrm{C} 2)$ is obtained.

\section{APPENDIX D: BEHAVIOUR OF THE NARROW GAP SOLUTION FOR HIGH SHEAR WAVE NUMBERS: WAVE EQUATION}

For high shear wave numbers the viscous forces can be neglected compared to the inertial forces. In this situation function $B(s)$ (see section 4.1 and Appendix B) can be approximated by

$$
B(s) \cong 1
$$

The polytropic constant $n$ reduces to $\gamma$ for high shear wave numbers. This means that the process occurs adiabatically (isentropically). A high shear wave number implies that viscous effects can be neglected. The "narrow gap" equation (B19) for the pressure perturbation $p$ now reduces to

$$
g^{2} \partial^{2} p / \partial x^{2}+(g / a)^{2} \partial^{2} p / \partial y^{2}+k^{2} p=-k^{2} \gamma h .
$$

This equation is a modified form of the wave equation. Equation (D2) can be derived directly from the wave equation. When the pressure is assumed constant across the gap width, small perturbations and dimensionless co-ordinates are introduced, and the resulting equation is integrated with respect to the gap width, equation (D2) is obtained. 
APPENDIX E: LIST OF SYMBOLS



